\title{
Identification of paired immunoglobulin-like type 2 receptor $\alpha$ as hepatitis B virus DNA polymerase transactivated protein 1 interacting proteins
}

\author{
YONG-ZHI LUN, QING CHI, XUE-LEI WANG, FANG WANG and WEN SUI \\ Liaoning Provincial University Key Laboratory of Biophysics, College of Medicine, Dalian University, \\ Dalian, Liaoning 116622, P.R. China
}

Received July 9, 2013; Accepted November 8, 2013

DOI: $10.3892 / \mathrm{mmr} .2013 .1813$

\begin{abstract}
Hepatitis B Virus (HBV) DNA polymerase transactivated protein 1 (HBVDNAPTP1) is a novel protein transfected by HBV DNA polymerase, which has been screened by a suppression subtractive hybridization technique. In the present study, a yeast two-hybrid system was used to screen the proteins interacting with HBVDNAPTP1 in leukocytes in order to investigate the biological function of HBVDNAPTP1. The HBVDNAPTP1 coding sequence was cloned into a pGEM-T vector. Subsequent to sequencing, the HBVDNAPTP1 was subcloned into the bait plasmid pGBKT7 and transformed into yeast AH109. Western blotting confirmed the presence of HBVDNAPTP1 expression in the AH109 yeast strains. The transformed yeast AH109 cells were mated with Y187 yeast cells containing the leucocyte cDNA library pACT2 plasmids in $2 \mathrm{X}$ yeast extract peptone $\mathrm{D}$-glucose adenine (YPDA) medium. For selection and screening, diploid yeast was plated on synthetic dropout medium (SD/-Trp-Leu-His-Ade) containing X- $\alpha$-gal. Following sequencing and the verification of the open reading frames of positive colonies, four different proteins were obtained. To further confirm the interaction between HBVDNAPTP1 and the screened proteins, paired immunoglobulin-like type 2 receptor $\alpha$ (PILRA), one of the positive colonies, was cloned. The glutathione $S$-transferase pull-down in vitro assay and a co-immunoprecipitation in vivo assay were used to examine the interaction between HBVDNAPTP1 and PILRA, respectively. HBVDNAPTP1 may be involved in the negative regulation of
\end{abstract}

Correspondence to: Dr Yong-Zhi Lun, Liaoning Provincial University Key Laboratory of Biophysics, College of Medicine, Dalian University, 10 Xuefu Street, Dalian Development Area, Dalian, Liaoning 116622, P.R. China

E-mail: lunyz@163.com

Key words: hepatitis B virus, DNA polymerase, transactivators, two-hybrid system techniques, paired immunoglobulin-like type 2 receptor $\alpha$ the PILRA-mediated Janus-activated kinase/signal tranducer and activator of transcription signaling pathway, and exert a positive effect on the initiation of monocyte apoptosis. These results contribute our knowledge of the biological functions of HBVDNAPTP1 and provide novel data to aid in the further analysis of the regulatory mechanism of this protein.

\section{Introduction}

It is well known that subsequent to the infection of hepatitis B virus (HBV) into the target cells, the interactions of the virus genome and proteins with the genes and proteins in the target cells are significant in determining HBV replication, immune evasion and chronic infection (1). In previous years, it has been revealed that a complex trans-regulatory mechanism is involved in the interaction of HBV with the target cells, and the HBV proteins are trans-regulatory in the gene expression of these cells (2). The critical antigen components of HBV associated with trans-regulatory function have been identified in target cells and the specific mechanisms have been clarified, which are of great significance in confirming the pathogenic mechanisms of HBV and identifying effective prevention and treatment methods. HBV DNA polymerase transactivated protein 1 (HBVDNAPTP1) is a protein that is worth studying. Thus, suppression subtractive hybridization technology (GenBank accession no. AY450389). has been used to study the trans-regulatory target genes of the HBV DNA polymerase, which was verified by dot blot hybridization. The HepG2 hepatoblastoma cell line was screened to obtain a novel gene (GenBank accession no. AY450389), which was located on the long arm of chromosome 9, region 2, band 2, sub-band 31 (9q22.31). With the use of the Unigene database (http://www.ncbi.nlm.nih.gov/unigene) for an expression analysis of tissue distribution, this gene was observed to be expressed in a variety of tissues, excluding the pituitary gland, tonsil, tongue, thymus, trachea and umbilical cord. A preliminary study clarified that HBVDNAPTP1 is localized in the cytoplasm (3). The yeast two-hybrid system is currently one of the most effective ways to study the function and proteins of novel genes. Thus, the present study mainly focused on the screening of HBVDNAPTP1 interacting proteins in order to reveal their biological functions. 


\section{Materials and methods}

Vector, strain and cell lines. The pGEM-T vector was purchased from Promega Corporation (Madison, WI, USA). The yeast cell expression vector, pGBKT7, and the AH109 haploid yeast cell line were obtained from the Infectious Disease Research Institute of Beijing Ditan Hospital, Capital Medical University (Beijing, China). The mammalian cell expression vectors, pCMV-Myc and pCMV-HA, were purchased from Clontech Laboratories, Inc. (Mountain View, CA, USA). E. coli DH5 $\alpha$, HepG2 hepatoblastoma and HEK293 human embryonic kidney cell lines were preserved in our laboratory (Dalian, China)

Reagents. TRIzol reagent and Lipofectamine PLUS were purchased from Gibco-BRL (Carlsbad, CA, USA). The DNA Glass-Milk Rapid Purification kit was purchased from BioDev (Milano, Italy). DNA marker, T4 ligase, Takara Ex Taq kit and restriction endonuclease enzymes EcoRI, BamHI, BgIII and SalI were purchased from Takara Biotechnology Inc. (Dalian, China). Yeast extract, tryptone and yeast nitrogen base were obtained from Oxoid (Hampshire, UK). Matchmaker GAL4 Two-hybrid system 3, pre-transformed Matchmaker libraries, yeast YPD medium, deficient amino acid mixtures and disulfuric acid adenosine were all purchased from Clontech Laboratories, Inc. The glutathione- $S$ transferase (GST) gene fusion system was obtained from Amersham plc, (Piscataway, NJ, USA). c-myc Tag monoclonal antibody, goat anti-mouse horseradish peroxidase (HRP)-IgG and the concentrated 3,3'-diaminobenzidine kit were all purchased from Zhongshan Golden Bridge Biotechnology Co., Ltd. (Beijing, China). Human influenza hemagglutinin (HA)-Tag monoclonal antibody, mouse IgG, Protein A+G Sepharose $4 \mathrm{~B}$ and the hypersensitive enhanced chemiluminescence kit were purchased from the Beyotime Institute of Biotechnology, (Haimen, China). Primer synthesis and DNA sequencing were performed by Takara Biotechnology Inc.

Amplification of the HBVDNAPTPl gene by quantitative polymerase chain reaction $(q P C R)$. Specific primers with EcoRI/BamHI sites were designed according to the HBVDNAPTP1 gene sequence from the National Center for Biotechnology Information: Primer 1: 5'-GAATTCATGATGTTTGTGCTGCTAAAC-3' and primer 2: 5'-GGATCCATAAGTCCTCTCTAAAATTGCG-3'. The total RNA from the HepG2 cells was extracted and transcribed into cDNA using oligo dT. The PCR amplification of the HBVDNAPTP1 gene was performed using the following conditions: $95^{\circ} \mathrm{C}$ for $5 \mathrm{~min}$, followed by 30 cycles at $94^{\circ} \mathrm{C}$ for $30 \mathrm{sec}, 55^{\circ} \mathrm{C}$ for $45 \mathrm{sec}$ and $72^{\circ} \mathrm{C}$ for $1 \mathrm{~min}$, and then $72^{\circ} \mathrm{C}$ for $10 \mathrm{~min}$.

Construction of yeast cell bait expression vector, $p G B K T 7-H B V D N A P T P 1$. The purified HBVDNAPTP1 PCR fragment was ligated into a pGEM-T vector and transformed into DH5 $\alpha$ competent cells. Positive colonies from X-gal/isopropyl $\beta$-D-1-thiogalactopyranoside selection were used for plasmid isolation. Following sequencing, the pGEM-T-HBVDNAPTP1 plasmid was digested by
$E c o \mathrm{RI} / \mathrm{Bam} \mathrm{HI}$, and the purified fragment was ligated into linearized pGBKT7 by EcoRI/BamHI and then transformed into DH5 $\alpha$ competent cells. The recombinant plasmid, pGBKT7-HBVDNAPTP1, was extracted by an alkaline lysis method and identified by enzyme digestion.

Transformation of AH109 yeast cells with pGBKT7-HBVDN$A P T P 1$. The AH109 yeast haploid cells were transformed with recombinant pGBKT7-HBVDNAPTP1 or pGBKT7 empty vector by the LiAc method, and spread onto synthetic dropout medium (SD)/-Trp filter plates. Positive yeast colonies were inoculated on synthetic defined/-Trp medium and agitated overnight at $30^{\circ} \mathrm{C}$. The yeast protein was extracted using the urea/SDS method. In total, $50 \mu$ of the extracted yeast protein was subjected to SDS-PAGE, transferred to a membrane and blocked with $5 \%$ skimmed milk overnight at $4^{\circ} \mathrm{C}$. The membrane was incubated with primary antibody (1:100; c-myc Tag monoclonal antibody) and secondary antibody (1:3,000; HRP-goat anti-mouse IgG). The expression of the bait protein, HBVDNAPTP1, was detected by 3,3'-diaminobenzidine chromogenic methods.

Yeast two-hybrid screening. The positive yeast colonies were screened according to the manufacturer's instructions for the yeast two-hybrid system (Clontech Laboratories, Inc., Mountain View, CA, USA). The yeast plasmid was extracted and transformed into DH5 $\alpha$ competent cells. The plasmid was extracted by the alkaline lysis method and identified by enzyme digestion. The positive clones (500-2,000 bp) obtained from enzyme digestion were verified further by sequencing. A homology analysis of the sequencing results was conducted by a basic local alignment search tool (BLAST) comparison (http://blast.ncbi.nlm.nih.gov/Blast.cgi).

Verification of candidate library vector. The extract containing the library vector of $E$. coli DH5 $\alpha$ and the AH109 yeast cells containing the pGBKT7-HBVDNAPTP1 bait vector were incubated in inverted quadruple dropout medium (QDO; $\mathrm{SD} /$-Ade/-His/-Leu/-Trp) plates at $30^{\circ} \mathrm{C}$ for 7 days. The yeast colonies were selected again and inoculated into QDO/X- $\alpha$-gal plates. pGBKT7-HBVDNAPTP1 with candidate library vectors was individually co-transformed into AH109 cells. The cotransformed library and the empty vector, pGBKT7, served as negative controls.

Interaction between HBVDNAPTPI and the paired immunoglobulin-like type 2 receptor $\alpha$ (PILRA) intracellular domain by immunoprecipitation. The eukaryotic expression plasmids pCMV-Myc-HBVDNAPTP1 and pCMV-HA-PILRA intracellular domains [the cytoplasmic domain of PILRA,(PILRACD)] were constructed and co-transfected into HEK293 cells in 6 -well plates. The cells were lysed by NP-40 lysis buffer. In total, $2 \mu \mathrm{g}$ HA-tag monoclonal antibody or mouse IgG was added to the cell lysates and incubated at $4^{\circ} \mathrm{C}$ overnight. The cell lysates were incubated with $30 \mu \mathrm{l}$ protein $\mathrm{A}+\mathrm{G}$ Sepharose $4 \mathrm{~B}$ beads (Beyotime Institute of Biotechnology) overnight. The beads were collected by centrifugation at 3,000 rpm for $3 \mathrm{~min}$ at $4^{\circ} \mathrm{C}$, washed three times with lysis buffer and then boiled with $1 \mathrm{X}$ SDS-PAGE protein loading buffer for $5 \mathrm{~min}$. In total, $20 \mu \mathrm{l}$ of the supernatant was separated by SDS-PAGE and 
transferred to a PVDF membrane. Following blocking with $5 \%$ skimmed milk at $4^{\circ} \mathrm{C}$, the membrane was incubated with primary antibody (1:200) and secondary antibody (1:5,000; HRP-goat anti-mouse IgG), and then developed using the chemiluminescence method. The co-transfection of HEK293 cells with pCMV-Myc-HBVDNAPTP1 and pCMV-HA was used as a negative control.

Interaction between HBVDNAPTP1 and the PILRA intracellular domain by GST pull-down. The prokaryotic cell expression plasmid, pGEX-4T-1-PILRACD, was constructed and transformed into BL21 (DE3) competent cells. The positive colony was inoculated overnight and scaled-up in a 1:20 dilution until the bacterial concentration reached $\mathrm{A}_{600}=0.6$. A final concentration of $0.5 \mathrm{mmol} / 1$ isopropyl $\beta$-D1-thiogalactopyranoside was added and cultured at $30^{\circ} \mathrm{C}$ for $4 \mathrm{~h}$. The bacterial cells were harvested, washed and suspended in $1 \mathrm{X}$ phosphate-buffered saline. Following ultrasonication (Sonics \& Materials, Inc., Newtown, CT, USA), the cell supernatant was collected subsequent to centrifugation. In total, $400 \mu$ l Glutathione Sepharose 4B (Beyotime Institute of Biotechnology) was added to the bacterial lysis supernatant and incubated at $4^{\circ} \mathrm{C}$ for $2 \mathrm{~h}$. The mixture was centrifuged at $1,500 \times \mathrm{g}$ (Beckman Coulter, Inc., Brea, CA, USA) at $4^{\circ} \mathrm{C}$ for $3 \mathrm{~min}$ to remove the supernatant, and the beads were washed with lysis buffer. The BL21 (DE3) competent cells transformed with pGEX-4T-1 were used as a negative control. The GST pull-down method was performed in the same way as aforementioned, but protein A+G Sepharose 4B cross-linked with HA-Tag monoclonal antibody was replaced by Glutathione Sepharose 4B cross-linked with the GST-PILRACD fusion protein.

\section{Results}

Construction of yeast cell bait expression plasmid $p G B K T 7-H B V D N A P T P 1$. Using cDNA from the HepG2 cells as the template, the 444-bp HBVDNAPTP1 gene was amplified and ligated into the yeast cell expression vector, pGBKT7, by TA cloning and then identified by EcoRI/BamHI and Bg1II/SalI digestions. Gene fragments of 444 and 319 bp were obtained (Fig. 1). This indicated that the HBVDNAPTP1 gene was accurately ligated into the pGBKT7 vector.

Expression of HBVDNAPTP1 in yeast cells. As the pGBKT7 vector has a $\mathrm{C}$-myc tag sequence, the expression of the fusion proteins was examined by detecting the C-myc tag. Subsequent to the yeast cells being transformed by pGBKT7-HBVDNAPTP1, one clear band was observed and its molecular weight was consistent with the expected size of HBVDNAPTP1 (Fig. 2), indicating that HBVDNAPTP1 was successfully expressed in the yeast cells.

Results of yeast two-hybrid screen and verification. The transformed yeast culture, AH109, and the library cultures were combined together to screen for genes that interact with HBVDNAPTP1 in the leukocyte library. In total, 15 positive colonies were selected (data not shown). Since the pACT2 library plasmid contained two BglII restriction sites, the BglII enzyme was used to release the leukocyte cDNA library frag-

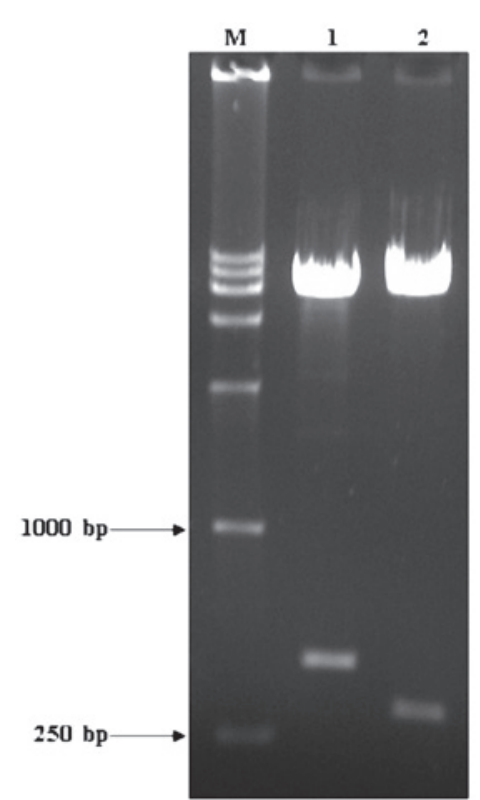

Figure1. Identification of pGBKT7-HBVDNAPTP1 by enzyme digestion. M, DNA marker DL15,000; 1, pGBKT7-HBVDNAPTP1 digested by EcoRI and BamHI; and 2, pGBKT7-HBVDNAPTP1 digested by $B g l \mathrm{II}$. HBVDNAPTP1, hepatitis B virus DNA polymerase transactivated protein 1.

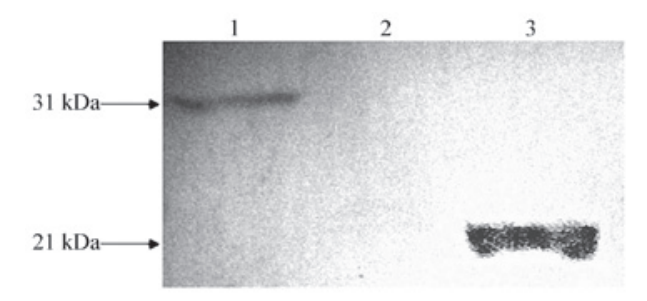

Figure 2. Identification of HBVDNAPTP1 by western blotting (yeast expression). 1, Cell lysates from pGBKT7-HBVDNAPTP1 transfected AH109 cells; 2, cell lysates from AH109 cells; and 3, cell lysates from pGBKT7 transfected AH109 cells. HBVDNAPTP1, hepatitis B virus DNA polymerase transactivated protein 1 .

ment, which was 400-2,000 bp in size. The plasmids from positive colonies were cut by $\mathrm{Bgl} I I$ and sent for sequencing. Using BLAST for the sequence comparison, four genes were obtained, including PILRA, zymogen granule protein 16, carboxylic acid lipase 1 and $\beta$ transduction element protein 2 , and their homology to the target genes was recorded as 98 , 100,99 and $98 \%$, respectively.

Interaction between HBVDNAPTP1 and the PILRA intracellular domain by co-immunoprecipitation. The full length of the PILRA protein is 303 aa and is composed of five domains; a signal peptide and the antibody variable, hinge, transmembrane and intracellular domains, of which the intracellular domain consists of 85 aa. Since the subcellular location of HBVDNAPTP1 is in the cytoplasm, it was speculated that it interacts with PILRA via the intracellular domain. The results of the present study demonstrated that the HA-PILRACD fusion protein could bind with the Myc-HBVDNAPTP1 fusion protein (Fig. 3). This result indicated that the intracellular domain of PILRACD is capable of specifically binding to HBVDNAPTP1 in vivo. 
$\mathbf{A}$

$\begin{array}{lll}\text { pCMV-Myc-HBV-PTP1 } & + & + \\ \text { pCMV-HA-PILRACD } & + & - \\ \text { PCMV-HA } & - & +\end{array}$

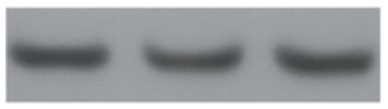

Myc-HBVDNAPTP1

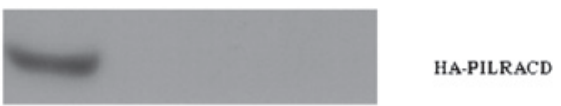

B

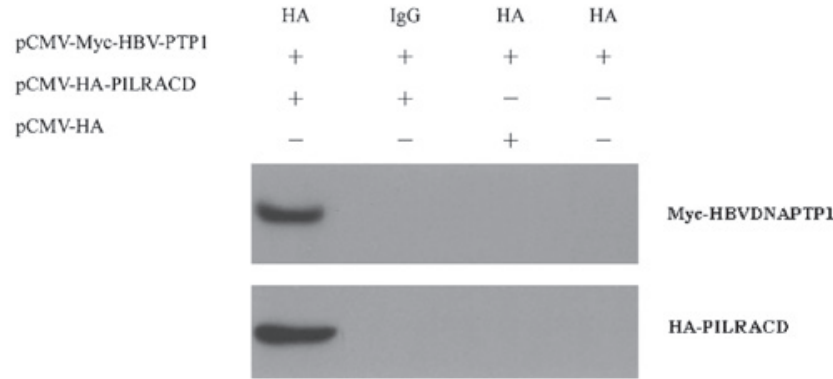

Figure 3. Interaction of HBVDNAPTP1 and PILRACD by an immunoprecipitation experiment. (A) Western blotting for whole-cell lysate samples and (B) western blotting analysis for immunoprecipitation samples. HEK293 cells were transfected with pCMV-Myc-HBVDNAPTP1 and pCMV-HA-PILRACD or pCMV-HA. Following cell lysis, cell lysates were immunoprepicitated with anti-HA antibody or IgG and blotted with anti-Myc or anti-HA antibodies. HBVDNAPTP1, hepatitis B virus DNA polymerase transactivated protein 1; PILRACD, paired immunoglobulin-like type 2 receptor $\alpha$ cytoplasmic domain; HA, human influenza hemagglutinin.

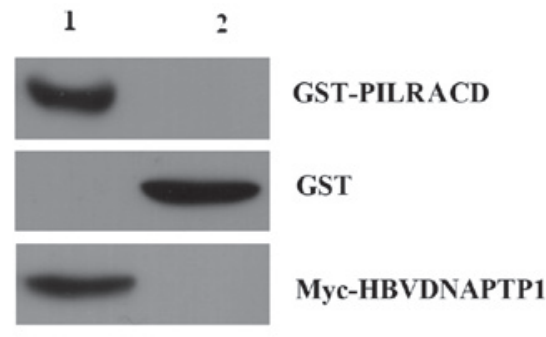

Figure 4. Western blotting for GST pull-down experiment. 1, Cell lysates from GST-PILRACD and Myc-HBVDNAPTP1 transfected cells; and 2, cell lysates from GST and Myc-HBVDNAPTP1 transfected cells. GST, glutathione S-transferase; PILRACD, paired immunoglobulin-like type 2 receptor $\alpha$ cytoplasmic domain; HBVDNAPTP1, hepatitis B virus DNA polymerase transactivated protein 1 .

Interaction of HBVDNAPTPl and the PILRA intracellular domain by GST pull-down. To verify the interaction of HBVDNAPTP1 and PILRA, GST-PILRACD fusion proteins and GST-tagged proteins were incubated with Glutathione Sepharose 4B, then incubated with the cell lysates of pCMV-Myc-HBVDNAPTP1 transfected cells. The GST pull-down experiment demonstrated that the GST-PILRACD fusion protein could bind with Myc-HBVDNAPTP1, but that the GST-tagged proteins were not capable of binding (Fig. 4). Thus, the GST pull-down experiment further validated the interaction of HBVDNAPTP1 and the PILRA intracellular domain.

\section{Discussion}

Chronic infection with HBV remains a worldwide health problem. The liver is the main target organ of HBV infection, but a number of extrahepatic tissues and organs, including the heart, spleen, lung and kidney, can also be infected by HBV (4-6). In previous years, various forms of HBV DNA, replicative RNA intermediates and antigen components have been detected in the peripheral blood mononuclear cells (PBMCs) of chronic HBV-infected individuals (7). The major clinical effect of the HBV infection of PBMCs is to cause host immune dysfunction, which leads to chronic HBV infection and causes latent chronic infection and mother-to-child transmission. Reinfection may occur following liver transplantation or using drugs to clear viruses from the serum $(8,9)$. Since HBV DNA can be replicated and transcribed by integration into the PBMC genome, the PBMCs have become significant replication sites and propagation vectors for HBV (10). PBMCs are mainly composed of lymphocytes, monocytes, granulocytes and other immunocompetent cells, which are supposed to be crucial in the body's immune response against HBV infection. However, HBV infection with PBMCs leads to cell dysfunction and a decline in the number of PBMCs, which indicates that HBV DNA replication and transcription in PBMCs cause cell apoptosis and inhibition of cell proliferation. Due to the inadequate $\mathrm{HBV}$-specific cellular immune response, the removal of the virus is difficult $(10,11)$.

Compared with the PBMCs of healthy volunteers, those of patients with chronic hepatitis B have upregulated expression of inducing apoptosis death receptor, FasL, and downregulated expression of resisting apoptosis decoy receptor, RAIL-R3 (12). Due to its antiviral and immunomodulatory effects, interferon (IFN)- $\alpha 2 \mathrm{a}$ is the drug treatment of choice for chronic hepatitis; however, IFN- $\alpha$ 2a cannot induce PBMC apoptosis and there is no significant change in the level of FasL expression in patients prior to and following treatment (12-14). There is a T-helper (Th)1/Th2 imbalance in patients with chronic 
hepatitis B. In patients with high levels of HBV DNA, the levels of interleukin (IL)-4 and IL-10 (Th2-type cytokines) in the peripheral blood are significantly higher compared with those in healthy volunteers, but the levels of IFN- $\gamma$ and IL-12 (Th1-type cytokines) are significantly lower. Th2 cytokines, particularly IL-4, can promote PBMC apoptosis in patients with chronic hepatitis B (14-15).

In the present study, a yeast two-hybrid system was used to screen a human leukocyte cDNA library, which was validated by intracellular co-immunoprecipitation. To the best of our knowledge, this study is the first to report that HBVDNAPTP1 interacts with the PILRA intracellular domain. PILRA is a transmembrane receptor with the effect of inhibitory regulation, which is not expressed in lymphocytes, but mostly in monocytes (16). The intracellular domain contains two immune-receptor tyrosine-based inhibition motifs (ITIMs). As PILRA binds with its ligand (primary cytokines), it is triggered to form homodimers and cause self-phosphorylation and activation of the non-receptor tyrosine kinase, Janus-activated kinase (JAK), which is followed by catalyzing the phosphorylation of tyrosine residues in ITIMs. At the same time, potential docking sites containing two Src homology 2 (SH2) domains of SH2-containing phosphatase 1 (SHP-1) tyrosine phosphatase are formed $(16,17)$. Once SHP-1 is docked at this docking site, the dephosphorylation of JAK and its downstream signaling molecules, signal transducers and activators of transcription (STAT), can be catalyzed, and thereby terminate the cell proliferation signal or induce an apoptosis signal $(18,19)$. It is of note to mention that in normal PBMCs, PILRA binds to cytokines, but does not terminate cell proliferation or trigger apoptosis. This means that PILRA requires a positive regulatory role to activate downstream signaling pathways. Due to the interaction of HBVDNAPTP1 and PILRA, it is easy to speculate that HBVDNAPTP1 is likely to bind with PILRA to mediate the negative regulation of the JAK/STAT signaling pathway. In the case of no ligand binding to PILRA, the downstream JAK/STAT signaling pathway cannot be activated. However, if a ligand binds to PILRA, the interaction of PILRA and HBVDNAPTP1 exhibits a positive role in the regulation, which can coordinate the activation of the downstream JAK/STAT signaling pathway, and thus activate the monocyte apoptosis signal. The low expression of HBVDNAPTP1 under normal conditions cannot effectively exhibit a positive regulatory role and cannot coordinate the activation of the JAK/STAT signaling pathway and induce apoptosis.

HBVDNAPTP1 interacting with the cytoplasmic domain of PILRA was examined successfully by GST pull-down in vitro and co-immunoprecipitation assay in vivo, respectively. HBVDNAPTP1 may be involved in the negative regulation of PILRA-mediated JAK/STAT signaling pathway.

\section{Acknowledgements}

The authors would like to thank the technical staff of the Institute of Infectious Diseases, Beijing Ditan Hospital, Beijing, China, for their excellent technical assistance.

\section{References}

1. Eyre NS, Phillips RJ, Bowden S, Yip E, Dewar B, Locarnini SA and Beard MR: Hepatitis B virus and hepatitis $C$ virus interaction in Huh-7 cells. J Hepatol 51: 446-457, 2009.

2. Cheng J: Trans-regulation mechanism in the pathogenesis of viral hepatitis. Shi Jie Wei Chang Bing Xue Za Zhi 11: 888-896, 2003 (In Chinese).

3. Lun YZ, Lei S, Cheng J, Wang YJ, Wang Q, Shen LT, Jiang HH and Zhang Y: Subcellular localization and structure prediction of HBVDNAPTP1 transactivated by hepatitis B virus DNA polymerase. Jie Fang Jun Yi Xue Za Zhi 34: 280-282, 2009 (In Chinese).

4. Chotiyaputta W, Pelletier SJ, Fontana RJ and Lok AS: Long-term efficacy of nucleoside monotherapy in preventing HBV infection in HBsAg-negative recipients of anti-HBc-positive donor livers. Hepatol Int 4: 707-715, 2010.

5. Lai M and Liaw YF: Chronic hepatitis B: past, present, and future. Clin Liver Dis 14: 531-546, 2010.

6. Pontisso P, Vidalino L, Quarta S and Gatta A: Biological and clinical implications of $\mathrm{HBV}$ infection in peripheral blood mononuclear cells. Autoimmun Rev 8: 13-17, 2008.

7. Lu L, Zhang HY, Yueng YH, Cheung KF, Luk JM, Wang FS and Lau GK: Intracellular levels of hepatitis B virus DNA and pregenomic RNA in peripheral blood mononuclear cells of chronically infected patients. J Viral Hepat 16: 104-112, 2009.

8. Coppola N, Pisapia R, Tonziello G, Martini S, Imparato M, Piai G, Stanzione M, Sagnelli C, Filippini P, Piccinino F and Sagnelli E: Virological pattern in plasma, peripheral blood mononuclear cells and liver tissue and clinical outcome in chronic hepatitis B and $\mathrm{C}$ virus coinfection. Antivir Ther 13: 307-318, 2008.

9. Gatta A, Giannini C, Lampertico P, Pontisso P, Quarta S, Zignego AL, Atzeni F and Sarzi-Puttini P: Hepatotropic viruses: new insights in pathogenesis and treatment. Clin Exp Rheumatol 26 (1 Suppl 48): S33-S38, 2008.

10. Hollinger FB and Sood G: Occult hepatitis B virus infection: a covert operation. J Viral Hepat 17: 1-15, 2010.

11. Bai GQ, Li SH, Yue YF and Shi L: The study on role of peripheral blood mononuclear cell in HBV intrauterine infection. Arch Gynecol Obstet 283: 317-321, 2011.

12. Hou W, Liu KZ, Li MW and Wo JE: Effect of IFNalpha-2a on Fas expression and apoptosis rate of peripheral blood cytotoxic $\mathrm{T}$ cells in patients with hepatitis B. Hepatobiliary Pancreat Dis Int 4: 403-405, 2005.

13. Karan MA, Oztürk S, Yenerel M,Erten N, Cefle K, Palanduz S and Tascioglu C: The in vitro effect of interferon-alpha 2a on CD95 expression of T cells in hepatitis B. Hepatogastroenterology 50: 2031-2034, 2003

14. Xing TJ, Zhang L, Luo KX, Hou JL, Zhang MX and Feng XR: Effect of cytokines on lymphocyte apoptosis in the patients with chronic hepatitis B in vitro. Med J Chin PLA 25: 16-18, 2000 (In Chinese).

15. Park Y, Park Y, Han KH and Kim HS: Serum cytokine levels in patients with chronic hepatitis B according to lamivudine therapy. J Clin Lab Anal 25: 414-421, 2011.

16. Fournier N, Chalus L, Durand I, Garcia E, Pin JJ, Churakova T, Patel S, Zlot C, Gorman D, Zurawski S, Abrams J, Bates EE and Garrone P: FDF03, a novel inhibitory receptor of the immunoglobulin superfamily, is expressed by human dendritic and myeloid cells. J Immunol 165: 1197-1209, 2000.

17. Mousseau DD, Banville D, L'Abbé D, Bouchard P and Shen SH: PILRalpha, a novel immunoreceptor tyrosine-based inhibitory motif-bearing protein, recruits SHP-1 upon tyrosine phosphorylation and is paired with the truncated counterpart PILRbeta. J Biol Chem 275: 4467-4474, 2000.

18. Saha B, Jyothi Prasanna S, Chandrasekar B and Nandi D: Gene modulation and immunoregulatory roles of interferon gamma. Cytokine 50: 1-14, 2010.

19. Xiao W, Kashiwakura J, Hong H, Yasudo H, Ando T, Maeda-Yamamoto M, Wu D, Kawakami Y and Kawakami T: Phospholipase C- $\beta 3$ regulates FceRI-mediated mast cell activation by recruiting the protein phosphatase SHP-1. Immunity 34 : 893-904, 2011 